EVS26

Los Angeles, California, May 6 - 9, 2012

\title{
HV Traction Battery: From Layout to Realization
}

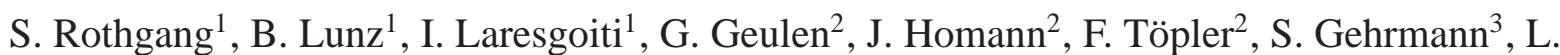 \\ Eckstein $^{2}$, and D. U. Sauer ${ }^{1}$ \\ ${ }^{1}$ Chair for Electrochemical Energy Conversion and Storage Systems, Institute for Power Electronics and \\ Electrical Drives (ISEA), RWTH Aachen University; Jaegerstrasse 17-19, D-52066 Aachen, Germany; \\ batteries@isea.rwth-aachen.de \\ ${ }^{2}$ Institute for Automotive Engineering, RWTH Aachen University \\ ${ }^{3}$ Air Energy GmbH und Co. KG
}

\begin{abstract}
From system layout to the vehicle integration a lot of design steps have to be taken into account regarding high-voltage (HV) batteries for electric vehicles. Electrical performance has to be traded off against mechanical and thermal issues several times during development. Besides the physical setup also the management of the battery system has to be considered and adapted to the chosen system layout, which leads among others to an optimized amount of sensors that are needed to ensure the safety of the system. As especially public transport is a promising sector for electric mobility, technical questions such as the thermal management and cell contacting shall be considered and shown by the example of a battery for a full-electric bus in the pilot-project "Smart Wheels", supported by the German Ministry of Economics and Technology (BMWi). Within the project the battery system as well as a fast charge concept for the whole day operation of the vehicle were developed and taken into operation. This work aims to give an overview of the full development cycle in the design of a new traction battery including test results of the prototype and solutions for technical questions such as the thermal management.
\end{abstract}

Keywords: battery, BEV, fast charge, public transport, thermal management

\section{Introduction}

Currently the industrial nations are responsible for around $60 \%$ of the worldwide $\mathrm{CO}_{2}$ emissions. The overall $\mathrm{CO}_{2}$ emissions in 2010 were $30.6 \cdot 10^{9}$ tons. The combined share of electricity, heat generation and transport represents nearly twothirds of the yearly global emissions with transport having a share of $23 \%$ in 2009 [1]. With the ongoing industrialisation of the developing countries and the related urban megacities and increased energy needs, the impact of these sectors and especially the transportation will even increase. Hence, it is especially important to reduce the emissions within these growing living areas which can be achieved by locally emissionfree individual transport. Benefits like using the bus lanes or paying less city toll are already provided by several cities like London to encourage people to drive electric cars and electric buses are mostly allowed to enter traffic-calmed inner cities. In addition to it, a global emission free transport could be realized using renewable energies only.

Nevertheless, only reducing emissions won't last as only justification for electric mobility as the ecological advantage over diesel becomes less important with more severe emission standards as EURO VI being introduced by 2013. These strict standards lead on the other hand to high efforts and costs for the car manufacturers to match 
the regulations for diesel propulsion that might close the financial gap to electric vehicles. This financial aspect is reinforced by the rising oil price. With 107 \$ per barrel as an annual average in 2011 the oil price reached the highest level ever [2].

As the driving profiles, ranges and route characteristics of a public bus are known exactly and a high mileage is ensured, electrification is especially promising and might offer chances to introduce electric mobility in the market. Moreover, it is possible to charge only at defined places without a need for a highly comprehensive public charging infrastructure. Hence, a roll out is possible relatively independent from infrastructural circumstances. To enable cost effectiveness it is crucial to guarantee a high number of operation hours and to optimize the battery in terms of operation and lifetime as it is the cost-dominating component in electric vehicles.

Based on the project "Smart Wheels" this paper discusses the full development cycle of a battery for a public bus regarding the reliability in operation and an optimal battery size in terms of lifetime, vehicle costs and operability. Moreover, a concept for the thermal management as crucial point for the aging of the cells and the operability of the battery is addressed.

\section{Design Steps}

The design of a large scale battery can be divided in three fundamental development steps, the demand analysis, the system design as well as the system production. During the first stage the dimensioning of the system as well as the battery technology and the cell types are addressed. In the following the system layout focuses on the overall design of the battery. Important steps as shown later in this work are the thermal management of the battery, the contacting of the cells as well as the mechanical stability and the crash safety. Moreover, the precharge of the intermediate circuit has to be considered and a parameterized battery management is needed. In the last stage the system is build-up and tested. During all steps safety and conformity to the regulations have to be assured.

\subsection{Demand Analysis}

Lithium ion battery cells are the most promising technology for electric mobility as they combine a high energy density with a high efficiency within certain limits. Therefore, cells with a high energy density are chosen for battery electric vehicles (BEV) and plug-in hybrid vehicles (PHEV) whereas high power cells are chosen for hybrid electric vehicles (HEV) or - depending on the needed $\mathrm{C}$ rate - for fast charge applications. Moreover, with around 5,000 full cycles lithium ion cells have a cycle stability superior to other battery technologies such as lead acid or nickel metal hydride. Nowadays, lithium ion cells are one of the most expensive battery technology and are responsible for $50-65 \%$ of the total battery pack costs, but due to mass production large economy of scale effects are expected. Therefore, it is estimated that the costs for nickel oxide (NCA) cells will decline by $60 \%$ to 270 330 \$/kWh [3].

Regarding the typical driving range of a public bus, an approximate range of 95 miles is needed. With 93 miles on an Aachen inner city line this is also true for the project Smart Wheels. Therefore, the energy consumption according to simulations would be around $120 \mathrm{kWh}$ which would lead to a battery size of at least 8501 and a weight of $1,100 \mathrm{~kg}$. As the specific vehicle in the project is a small inner city bus with an admissible total weight of only $5650 \mathrm{~kg}$ the battery itself would reduce the payload tremendously. Hence, reenergizing during operation is necessary by all means. To reduce weight and costs of the system it was decided to limit the energy content of the battery to $45 \mathrm{kWh}$ and to fast charge in between the rounds with a $60 \mathrm{~kW}$ charger. Two different charging strategies can be considered:

- discharging the whole battery and recharging via fast charge in a longer break or

- partly discharging the system and recharging every other round.

Deciding on the strategy, aging has to be taken into account. It can be divided between calendric aging and aging due to cycling whereas both lead to an increasing internal resistance and a decreasing available capacity. Calendric aging effects are parasitic side reactions in the cell that are strongly dependent on cell voltage and temperature. Cycling has an impact on lifetime due to voltage displacement, temperature rise and mechanical stress inside the cell [4]. Mechanical stress is caused by volume changes of the active material due to lithium (de-)intercalation and therefore dependent on the cycle amplitudes. Deeper cycles lead to a stronger aging. Hence, a reduction of the used capacity range by charging more often can limit aging albeit the overall charging time per day remaining the same [5]. Therefore, it was decided to charge the battery every other round for maximum 15 minutes which leads to a stepwise declining SOC during the day as shown exemplarily by the simulation in Fig. 1. Even though the overall energy consumption in the simulation is $77 \mathrm{kWh}$, the single cycles are only $15.4 \mathrm{kWh}$ and the maximum overall discharge amplitude is limited.



Figure 1: Simulation of a full day driving cycle with a fast charge every other round 
Due to safety reasons, it is essential to ensure a failure-free operation of the battery in mobile applications. A shutdown of the battery system is the ultimate possibility that should only occur as last choice, as it might happen in a crucial driving situation. Examples for that are turning left, crossing railroad tracks or when the active moment distribution is activated. In these situations a shutdown of the battery might lead to a higher safety risk as the fault in the battery itself. Therefore, it was decided to divide the battery in two packs that are directly in parallel to minimize the risk of complete failure as an unmanageable fault would have to occur in both packs at the same time.

Regarding the axle weight distribution and the available constructed space it was decided to take pouch bag cells as they have the best energy to volume ratio. Due to the vehicle requirements a working voltage between $200-450 \mathrm{~V}$ was needed. That leads to a series connection of 98 cells with a nominal voltage of $3.7 \mathrm{~V}$. To achieve the desired energy content a capacity of at least $60 \mathrm{Ah}$ per battery pack was needed. Due to the relatively high load during the quick charge, a high power cell was favored. The agglomeration of the cumulative decision criteria brought forth the choice of the SLPB110255255H, a 60 Ah high energy cell of Kokam [6].

\subsection{System Design}

After having chosen a cell type and decided on the general system layout in the demand analysis, the system design addresses the specific layout of the battery packs including the cell contacting and the overall mechanical setup as well as the battery management system.

For the housing of the battery it is essential to take the mounting position into account. On the one hand cells have to be protected against mechanical impact during a crash by all means, on the other hand it has to be guaranteed that the battery packs don't cause further damage in the passenger cabin. Moreover, the weight distribution of the vehicle before the conversion to electric propulsion has to be considered as it should only be changed within certain limits. This leads to the conclusion that the packs can either be installed on the underfloor or on top of the vehicle, with the latter affording the bigger changes in the vehicle structure. In the approached city sprinter an underfloor-installation outside the crashzones of the vehicle behind the two axes was chosen. This limited the available battery space to $870 \times 580 \times 360 \mathrm{~mm}$ each and required strong vehicle-mounted ground plates to protect the battery against damage due to e.g. speed bumps. The housing itself consists of $4 \mathrm{~mm}$ thick aluminum U-profiles and a ground plate that is also used as cooling plate as shown later in this work. For waterproofness the housing is welded and the lid sealed with a gasket and it is made sure that according to IP $4 \mathrm{X}$ no contact to active parts is possible to protect people against electric shock [7]. Therefore a specified HV-plug for the automotive sector was needed.
This completely sealed housing would also prevent the battery from emitting gas. Cell failures as a thermal runaway normally go along with an overpressure within the cell. Due to that overpressure pouch bag cells vent as the vacuumed foil opens next to the cell contacts. An 18650 cell of only around $2.6 \mathrm{Ah}$ emits approximately 2.51 of gas [8]. Therefore, it is essential to add a pressure vent in the housing of the battery as well. The pressure vent consists of a membrane that is jammed in between two aluminum plates which are screwed to the housing, as shown in Fig. 2. Test results show that the membrane is blown out by a pressure of 1.0-1.2 bar.

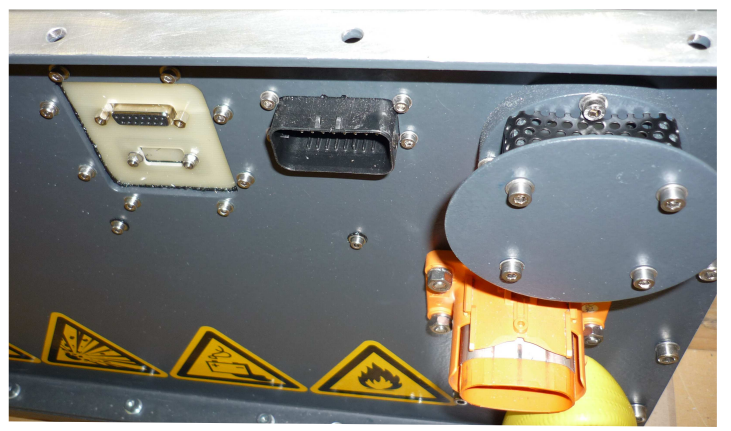

Figure 2: Front of the housing with the IP compatible $\mathrm{HV}$-plug and the vent at the right hand side

Having the highest energy density, pouch bag cells on the other hand go along with the disadvantage of the lowest mechanical stability. To prevent the cells from damage, they have to be stabilized in the designated position. Therefore, the pack was divided into 8 equal sections by aluminum partition walls. The 98 cells were divided in 7 modules of 14 cells each, the remaining compartment houses the switch box and the electronics.

In each module the cells are interconnected alternating by two copper plates that fixate the contacts with screws in between. The copper plates are constricted with a rubber strip that fixates the screws and ensures insulation. The modules were wrapped in rigid foams to pad the cells and to enable a slight expanding of the cells. It is essential to use only self-extinguishing materials inside the pack to prevent a single cell fault to inflame further cells. The final layer of the modules shown in Fig. 3 is Teflon foil as insulation. The sections itself are also lined with Teflon. This insulation is important, as the constant vibration in a vehicle might lead to a fraying of the cell foil and consequently to an insulation fault.

The bottom area of the sections is coated with a soft silicone foil. The material has a high flexibility to enhance the connection to the cooling system and ensure at the same time an outstanding electrical insulation. At this juncture a trade off has to be found as good electrical insulation normally does not go along with a good thermal conductivity. However, the insulation is of particular importance in that case, as there are - as explained hereafter in section 4.2 - aluminum plates installed in between the cells due to cooling. A direct contact of these plates to the 
ground plate would basically equal a huge capacitor which has to be prevented in the vehicle due to EMC issues.

The battery is monitored by the battery management system of Air Energy, that is divided in a master-slave architecture. Each module is monitored by a slave that interacts with the joint master. A joint master is necessary as the two packs are directly parallel-connected and thus the interconnecting of the packs has to be prevented if they have a different voltage level. Different voltage levels can occur due to e.g. self discharge after a longer downtime and would lead to inadmissible high compensating currents. The measured voltages and temperatures of each cell are $\mathrm{A} / \mathrm{D}$ converted at the slaves and sent to the master. The master controls the precharge and interconnecting of the packs and sets, as the communication interface to the vehicle, the maximum load levels for the charge or discharge procedure. The master controls the adherence of the threshold values and can either derate the load or shutdown one of the packs. As a fall back level for a malfunctioning master, the slaves can shutdown the pack directly via a safety circuit if a second threshold is exceeded. As already the A/D conversion for the safety circuit is done independently a complete redundancy is given.

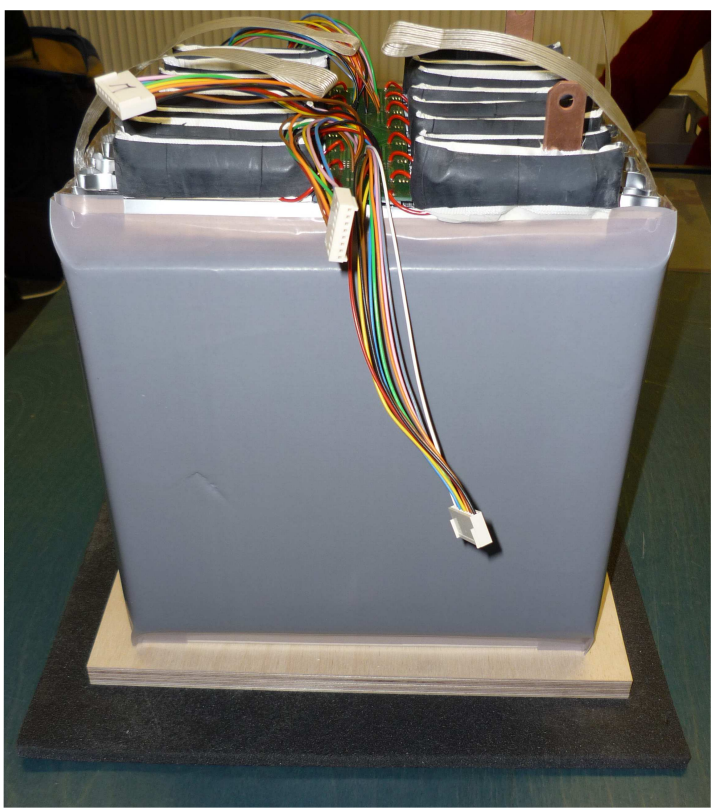

Figure 3: Module of 14 cells with the contacts screwed together and a Teflon insulation (constructed by Air Energy)

The battery system can be disconnected from the rest of the drive train by relays at both poles. The relays are normally open which is the safe state of the system. During driving operation they have to be held shut actively. Additionally to the switches the battery pack is protected against overcurrent by two DC-fuses and the pack current is measured. Those melting fuses with a nominal value of 200 A ensure a protection against external short-circuits.
While the front battery pack contains the master as shown in Fig. 4, the rear pack houses the insulation monitoring device that can monitor the complete electric drive train. To enable driving operation with a shutdown rear pack, the insulation monitoring device has to be placed at the drive train side of the relays. This requirement is motivated by the high risk that goes along with a complete system shutdown due to the particular driving situation.

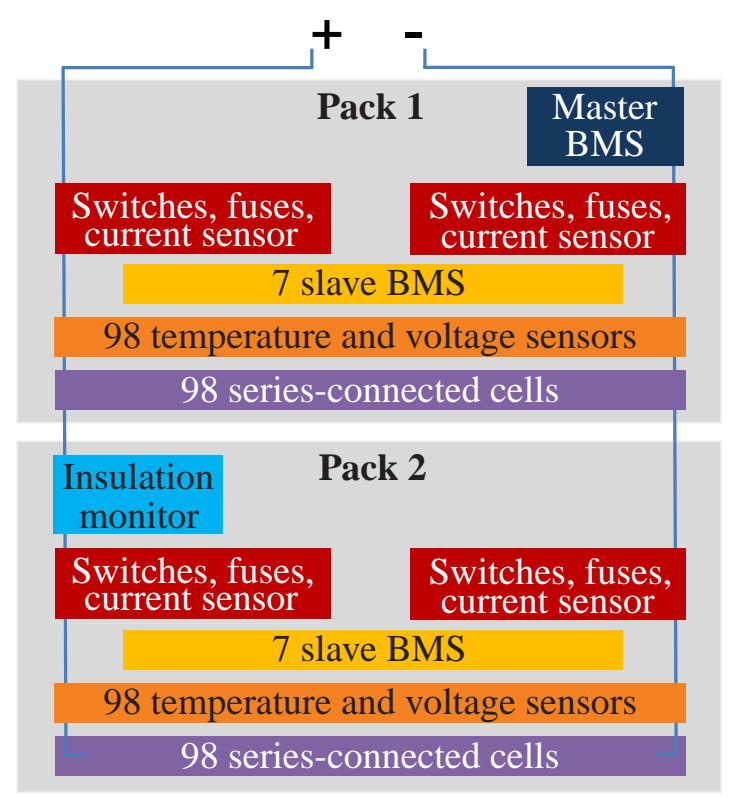

Figure 4: Schematic of the system layout

Regarding the voltage measurement, it is indispensable to monitor every single cell voltage in a series connection. Overcharging a cell is one of the highest safety risks as it might lead to a thermal runaway. A thermal runaway is an exothermal process that is initiated by a trigger event like e. g. an overcharge or a deep discharge. The evaporated heat in turn accelerates the exothermal process due to the decomposition of the active material. Hence, the highest voltage in the string limits the charging and the other way round. Voltage inequalities can be caused by aging or different temperatures and due to this different internal resistances of the cells. Therefore, it is important to balance the cells, which is realized by a passive balancing system.

Additionally to the cell voltages the cell temperatures were measured by a temperature sensor in the area in between the positive and negative contact. Measurements have shown, that the warmest spot of the cell is in the upper cell area on the middle axis. By installing a sensor at that position in the module and not directly on a cell surface, each cell temperature is measured twice with a slight time delay and therefore a redundancy against a sensor failure is given.

As the pack was produced for a prototype bus and academic research it was of great interest to monitor all cell temperatures in detail. However, installing a sensor per cell would be too expensive for series production. Therefore, it was analyzed 
what aftermaths a single cell fault has and how many sensors are really needed to detect it. For this purpose a module was simulated with Comsol Multiphysics. In the simulations it was estimated that the sensor is in a thin block of air as it has no direct contact to the cell surface. The volume-related power losses of the cell

$$
\begin{aligned}
Q_{\text {nom }} & =\frac{P_{\text {losses }}}{V_{\text {active }}} \\
& =\frac{8.17 \mathrm{~W}}{51.94 \cdot 10^{4} \mathrm{~mm}^{3}} \approx 15729.69 \frac{\mathrm{W}}{\mathrm{m}^{3}}
\end{aligned}
$$

could be calculated by the measured internal resistance of the cells and therefore the overall losses of the cell $P_{\text {losses. As the pack itself has }}$ a cooling plate the ground plate was simulated with a constant temperature of $20^{\circ} \mathrm{C}$ and it was estimated that the module is thermally isolated as it is packed in hard foam.

To simulate single cell faults the losses of the cells were risen one by one while the rest of the cells in the module produced the standard thermal dissipation loss $P_{\text {losses. }}$. The increased losses $P_{\text {fault }}$ were set to $20,4 \mathrm{~W}$, as this causes temperatures above $60^{\circ} \mathrm{C}$. In Fig. 5 it is shown exemplarily for the cells 7 and 14. Those are the extreme cases due to their position in the module. It can be seen that a fault in a middle cell is cooled better due to the connection to the neighboring cells. At the same time, it is shown that a fault definitely will be detected by the directly adjoining sensors.

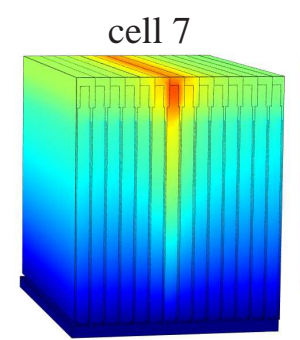

cell 14

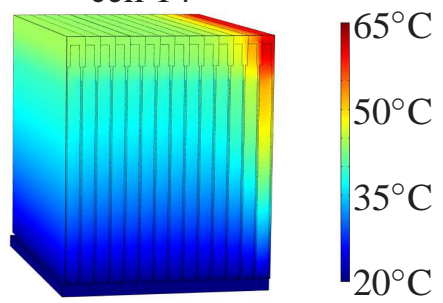

Figure 5: Temperature distribution for a single cell fault in cell 7 on the left side and cell 14 on the right side

Having shown that a cell fault can be detected with one sensor per cell, it is of interest to see whether or not a safe system operation is still possible with faulty sensors. Therefore, a single cell fault at cell 6 was simulated. The adjoint sensors 4, 5, 6 and 7 were assumed as out of work and therefore gave no or a misinformation about the cell temperatures. In Fig. 6 is shown, that a temperature difference in the module can be detected by a comparison of the temperature values of the sensors 3 and 8 with the lowest measured temperature in the pack. Therefore, the distance to a functioning sensor should not be more than two cells as it is otherwise not possible to get a measurable significant temperature gradient of 2 to $4{ }^{\circ} \mathrm{C}$. Moreover, it is essential for a save detection that the farthest sensor is working properly to measure the temperature gradient. According to this it might be more severe if the faulty sensors are evenly distributed. A sensor is excluded from the measurement if it is not reacting or sending implausible values three times in a row as otherwise a technically fully functional sensor might be excluded premature. A measured temperature above $50^{\circ} \mathrm{C}$ leads to a warning message to the vehicle and a reduced provided output power. If $55^{\circ} \mathrm{C}$ are exceeded the master shuts down the affected pack or if this does not work the safety circuit will ultimately open the relays at $65^{\circ} \mathrm{C}$.

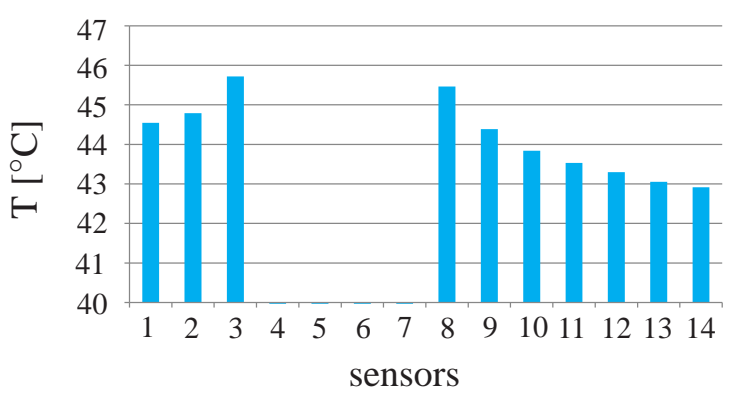

Figure 6: Measured temperatures of the sensors in a module with a single cell fault in cell 6 and defect sensors 4,5,6,7

Next to the supervising of the operation, the master also has to control the start up of the battery. If the relays of the battery would be closed without a start up procedure the input capacitors of the converter would be damaged by the inrush current as the load capacitance is charged up to the battery voltage. Moreover, the main fuse won't carry the inrush current and the relays would be damaged as well. Last but not least the cells are also not rated for the inrush current. Therefore, a precharge circuit is needed that consists of a precharge resistor $R_{\text {prech }}$ to limit the inrush current in series with a precharge relay and the main relays at both poles that ensure the safe state of the battery during downtime as both poles are disconnected. The precharge resistor is usually connected to the positive pole of the battery and needs to be dimensioned based on the capacity of the load and the desired precharge time. The current reaches $\frac{1}{e}$ of its initial value after the period

$$
T=R_{\text {prech }} \cdot C
$$

and has dropped to a manageable value after $5 \cdot T$. The energy

$$
E=\left(C \cdot U_{\text {bat }}^{2}\right) / 2
$$

that can be stored in the load's input capacitors $C$ has to be dissipated by the precharge resistor. Hence, the power dissipated by the precharge resistor during precharge is that energy over the precharge time

$$
P=\frac{E}{5 T}
$$

Though, the instantaneous power

$$
P_{\text {immediate }}=\frac{U_{\text {bat }}^{2}}{R_{\text {prech }}}
$$


the precharge resistor has to dissipate is a lot higher and due to this a high power precharge resistor is needed, even though it won't need to dissipate any significant power during overall operation. Yet, as most available resistors have a distinctly higher peak power dissipation, downsizing is possible as admissible precharge periods are below one second. Hence, a cooling of the resistor is also not needed. As many components are susceptible to the inrush current if the precharge fails, the proper performance of the precharge circuit should be checked before the main relays are closed. This can either be done by auxiliary contacts at the relays or measuring the voltage difference of the battery and the drive train.

\section{System Production}

Several regulations like e.g. the ECE R100.01 or the ISO 26262 have to be fulfilled during the system layout and also the system production. The goals are to protect people against electric shock and to enable a safe and reliable operation of the vehicle later on.

The protection against electric shock has to be assured on the one hand at the battery box but on the other hand also inside the battery system. Therefore all enclosures and barriers, which, when removed expose live parts of high voltage have to be marked with a symbol for HV. Moreover, the electrical parts have to be engineered and constructed in a way that insulation faults are prevented. Therefore, also the leakage distance as well as the distances due to insulation material have to be fulfilled. In the event of a battery fault including flames it is essential that there is as little as possible flammable material in the pack. Especially outer insulation parts and insulation parts that keep active parts in their designated position have to be sufficiently thermostable and resistant against ignition and fire spread to prevent a knock-on effect. During the commissioning the insulation resistance of the pack has to be measured regarding the dielectric strength and electric safety.

Moreover, the hardware components and the master-slave communication have to be checked during the system production. A representative amount of sensors has to be tested on accurate operation and it has to be ensured that they are mounted in the designated position. Moreover, the wiring of the boards and sensors has to be controlled. The boards have to be fixed tightly in the pack because of the high strain due to vibration in the vehicle and have to be housed EMCsafe by all means. The proper functioning of the BMS has to be tested during the initial startup. This includes a test of the correct reaction to all threshold values.

\section{Thermal Management}

In vehicle applications with on the one hand a high power requirement such as sport cars or ve- hicles with a fast charge application and on the other hand a tight packaging space an active cooling of the battery is mandatory. It is important to keep in mind that a cooling always has to be designed for the worst-case scenario and an aged cell. The worst case scenario is a hot summer day with the highest admissible power requirement of the vehicle the battery was designed for. An aged cell has a higher internal resistance and therefore higher thermal losses as a new one. A cooling can be done either with a cooling fluid or air and is either dependent or independent from the cooling system of the rest of vehicle.

\subsection{Necessity of a Thermal Management}

To analyze the necessity of a thermal management for the designed pack, a cell was cycled at the test bench with limited heat dissipation by the surface and without an acitve cooling. To thermally isolate the preheated cell, it was housed in styrofoam and four temperature sensors were attached to the cell surface at one side. One sensor was positioned in the lower part of the cell near at the middle axis, the sensors 2 to 4 in the upper area at the two sides and the middle axis. The cell was cycled with a $1 \mathrm{C}$-rate.

In Fig. 7 is shown that in the upper middle area in between the two contacts most of the heat is set free. After less than one complete dischargecharge cycle the cell has already reached the predefined threshold value of $50{ }^{\circ} \mathrm{C}$ and the experiment was interrupted. The relatively fast cooling rate indicates that the cell is by no means fully isolated. Thus, in the real operation the housing has a high thermal capacity and emits heat to the ambiance so that it can be assumed that the tested cell is isolated more than in the real operation in the module. Still it is unquestionable that a cooling is needed, as the experiment was run with a new cell and the load with $1 \mathrm{C}$ is less than in reality due to the fast charge. Moreover, a significant temperature difference could be detected in the cell which should be diminished by the cooling as well.



Figure 7: Cell surface temperatures of a thermally isolated cell during a $1 \mathrm{C}$ charge/discharge process 


\subsection{Thermal Connection of Cells}

Due to the large surface and the thin housing, pouch cells are superior to other cell geometries regarding the cooling. The upper area of the cell in between the contacts being the warmest area of the cell, it is important to dissipate the heat from there as well. As a heat dissipation in the chosen module design is only possible via the ground plate, an aluminum plate of $1 \mathrm{~mm}$ thickness covering the whole surface of the cell was needed in between the cells to ensure an evenly cooling within the cells. This effect is intensified by the close packaging, as the other side of the cell is pressed against the aluminum plate of the next cell. The cells were connected to the aluminum plate with a silicone to compensate surface irregularities, as it is essential to guarantee that there is no air in between as this would impair the cooling considerably. In Fig. 8 a cell with a detached aluminum plate is shown. The structure of the compensated cell surface irregularities can clearly be seen in the silicone layer. Still, small bubbles indicate air locks in between cell and aluminum plate. That shows that even though the silicone was carefully applied a complete coverage was not achieved. Therefore, it is essential to really apply a well-defined amount of silicone and to groud the two parts with an even pressure among the surface.

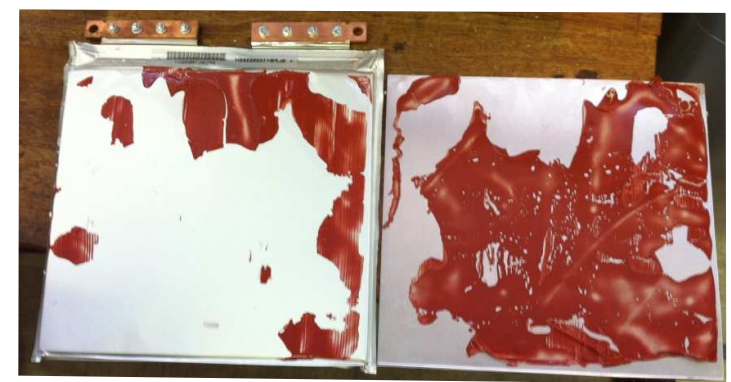

Figure 8: Cell and detached aluminum plate with the silicone layer indicating cell surface inequalities and air locks

\subsection{Homogeneity of the Temperature Distribution}

Due to aging it is important to get an as even as possible cooling, as the temperature is one of the crucial factors for calendric aging [9]. To design the optimized cooling, several factors have to be taken into account:

- available delivery rate of the pump,

- minimum pressure drop in the cooling system,

- minimum temperature gradient within the cells and

- minimum temperature gradient within the battery pack.
Within the vehicle only a relatively small pump was available that has a working curve below 0.15 bar. Moreover, the cooling fluid itself was only recooled by the ambient air. Therefore, a cooling system with the available pressure should be designed for an ambient temperature of $30{ }^{\circ} \mathrm{C}$ with a average heat generation of $0.26 \mathrm{~W} / \mathrm{cm}^{2}$ at the surface of the cold plate. This leads to a total heat influx $Q$ to the cold plate of $1161 \mathrm{~W}$. To find the optimal cold plate by a Comsol simulation the velocity

$$
v_{w}=\frac{w}{A}
$$

with the water flow

$$
w=\frac{m_{w}}{\rho}=\frac{Q}{c_{p} \Delta T \rho}
$$

and the cross-section A of the cooling channel is needed. The velocity is dependent on the admissible temperature gradient $\Delta T$ between inlet and outlet and the specific heat capacity $c_{p}$. Knowing the velocity, the Reynolds number to choose the right differential equation in Comsol can be calculated by

$$
R e=\frac{\rho v_{w} D_{h}}{\mu}
$$

with the hydraulic diameter $D_{h}$, the density $\rho$ of the cooling fluid and the dynamic viscosity $\mu$. To enable an as even as possible cooling it was decided to focus on a bifilar cooling as shown in Fig. 9. Those spiral windings are for example also used for underfloor-heating where an even temperature distribution is a prerequisite as well. The plate was build out of a thick aluminum plate with the windings milled into it and a covering lid of $3 \mathrm{~mm}$ thickness. To add an additional air gap of $2 \mathrm{~mm}$ in between the windings, which have a diameter of $15 \mathrm{~mm}$, was simulated but scrapped as the further decoupling of inlet and outlet only improved the temperature distribution by $0.2^{\circ} \mathrm{C}$ at an associated reduction of the available adherent of the two half's of the cold plate.

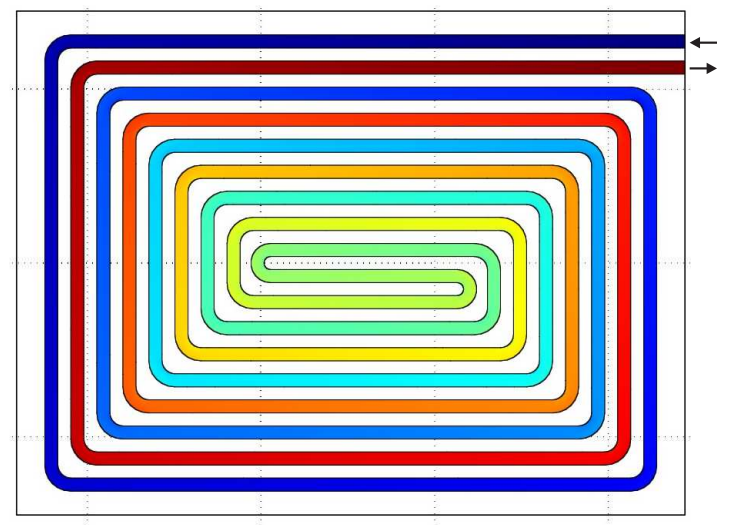

Figure 9: Bifilar cooling structure with the cool inlet (blue) and the hot outlet (red). 
As channel depth $4 \mathrm{~mm}$ to $6 \mathrm{~mm}$ were simulated with an inlet velocity of $0.6 \mathrm{~m} / \mathrm{s}$ to $0.8 \mathrm{~m} / \mathrm{s}$. As shown in Fig. 10 a channel depth of $6 \mathrm{~mm}$ and an inlet velocity of $0.7 \mathrm{~m} / \mathrm{s}$ are the optimal cooling option and with a pressure drop of 0.09 bar still admissible. With the optimized cold plate layout a temperature gradient below $3.5^{\circ} \mathrm{C}$ was achieved and the temperature gradient within the cell could be reduced to $2.5^{\circ} \mathrm{C}$ by the aluminum plates in between the cells.

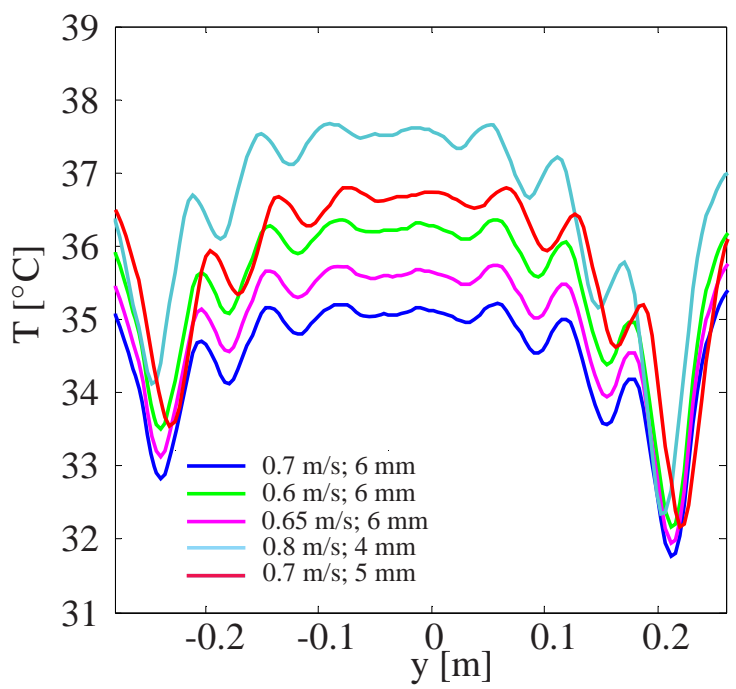

Figure 10: Temperature profile for different channel depths and velocities. The profile is a cross section along the $y$ axis in the middle of the cold plate on top of the aluminum cover.

\section{Test Results}

Finally a few results of the designed and build battery pack shall be shown. During the initial start up first of all the insulation resistance of the system was measured to ensure electric safety. Hence, a test voltage of $1000 \mathrm{~V}_{\mathrm{DC}}$ was applied for one minute according to ECE R100.01 between each pole and the housing. The measured insulation resistance has to be above $1 \mathrm{M} \Omega$ which was clearly exceeded for the tested packs, as it can be seen in Table 1. The following test on dielectric strength with $2.6 \mathrm{kV}$ was also succeeded without any disruptives.

Table 1: Insulation resistance [M $\Omega]$

\begin{tabular}{ccc}
\hline & Pack 1 & Pack 2 \\
\hline positive pole & 28.3 & 29.8 \\
negative pole & 50.4 & 49.1 \\
\hline
\end{tabular}

In the operation on the test bench the system was tested on the reaction to all threshold values. For all threshold values the warning messages and the correct opening of switches were proven. Moreover, a pulse test with the maximum admissible load was run and the procedure to connect packs with a differing voltages was tested. Having different voltages a reconnection is only possible during the charge process. The pack with the lower voltage level is charged until the voltages differences declined below a threshold value. In this moment the second pack is engaged and only minor compensating currents occur.

Additionally to the behavior of the battery management system, the cooling system was tested by simulating a full day operation. In Fig. 11 the measured daily cycle is shown. The system was discharged with the calculated mean discharge power for one hour and afterwards always fast charged for 15 minutes with $150 \mathrm{~A}$. As the temperature rise was relatively low due to the cold ambient temperature during the test and the new cells with a low internal resistance the battery system was heated purposefully with a 150 A charge-discharge current after 4.5 hours of operation. On reaching a temperature above $35^{\circ} \mathrm{C}$ the cooling of the second pack with a cooling fluid of $30{ }^{\circ} \mathrm{C}$ was started whereas the first pack remained uncooled. It can be seen that the temperature of the second pack heats on for a short time until it reaches a steady value whereas the first pack heats on until the system shuts down due to overtemperature. The temperature gradient within the cooled pack is only $2.5^{\circ} \mathrm{C}$ and therefore even better than the calculated $3.5^{\circ} \mathrm{C}$ maximum gradient.

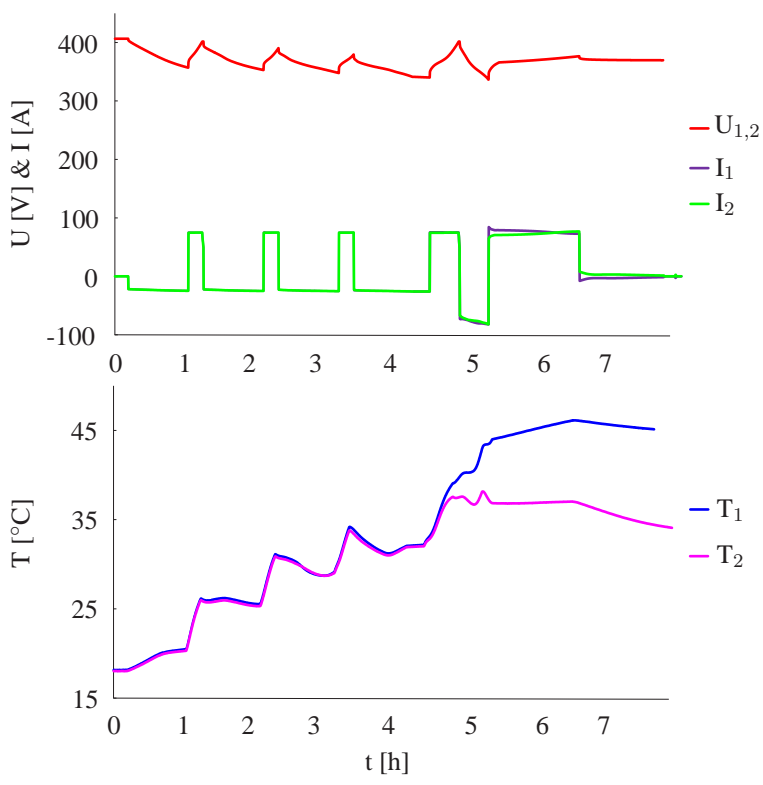

Figure 11: Daily cycle with a verification of the cooling concept by heating the system purposefully with a charge and discharge current of $150 \mathrm{~A}$ after 4.5 hours of operation and cooling only one of the packs in the following.

Having succeeded in all test bench experiments, the system was taken into operation in the vehicle and tested in real conditions. Moreover, the fast charge was successfully tested.

Finally an overview of the weight ratio of the components shall be given as the potential to 
reduce weight is of great importance for series production. Each of the packs weights approximately $215 \mathrm{~kg}$ with a cell weight of $149 \mathrm{~kg}$. Therefore, $66 \mathrm{~kg}$ are added to the weight necessary for providing the propulsion power. As it can bee seen in Fig. 12 the switches and monitoring system are negligible as there are three predominant weight adding factors. The cooling system with the ground plate and the 98 aluminum plates in between the cells weighs $23 \mathrm{~kg}$ whereby $80 \%$ are the aluminum plates. Therefore, a direct cooling of the contacts could reduce the weight significantly. An even bigger potential can be seen in the weight of the housing. As the modified vehicle was a conversion design vehicle, the battery had to be underfloor-installed and is therefore designed solid with an overall weight portion of $10 \%$ due to the crash safety. In a purpose design vehicle the system could be integrated in the floor directly to reduce weight. Finally the cell contacts could be welded instead of screwed together. This would reduce the weight by approximately $4-5 \%$ even if still a structure to keep the contacts in place would be needed.

- Housing
Contacting
BMS
Cells
Cooling
Switch Box
Rest

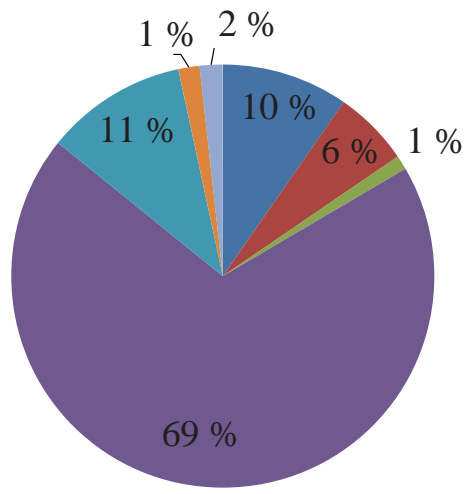

Figure 12: Weight ratio of the components

\section{Discussion}

It could be shown that the costs and size of a battery for public buses can be significantly reduced by using a smaller battery and fast charging every other round. The consequently smaller maximum driving range due to the smaller energy capacity does not limit the operation ability of the vehicle as the charging breaks are relatively short and can be done at the final stop of the vehicle. By recharging every round the charging time could even be reduced to less than ten minutes. Moreover, the charging time can be used at least as a small buffer time in operation for slight delays. With this approach electric mobility might in the future not only be a nice to have option for public transportation due to its local emission-free operation but competitive to diesel fuel driven buses that are nowadays state of the art. Contrary to private electric mobility the cycle life of the battery can completely be exploited and therefore a higher cost efficiency is achievable. The cells for a mobile battery system in general have to be chosen based on the estimated driving range, the needed load factors and the actually usable cycles. All of these determining factors are known in detail for public buses. Therefore, it is possible to choose the optimal cell for the respective application and to decide on a fundamental system structure. In the system structure especially the reliability of the system should be taken into account due to safety reasons.

The design steps in the system design can be divided in mechanical and electrical ones. Mechanical issues are the stabilization of the cells as especially pouch cells are quite flexible and the crash safety. It was shown exemplarily in the project Smart Wheels how this can be realized in a conversion design vehicle. Especially the housing could be realized lighter for a purpose design vehicle and also the cell alignment in modules could be modified for a more flexible construction space. Electrical issues are the insulation for electric safety and the management system that is usually divided in a master-slave architecture, which can include a fall back level. Regarding the measurements it can be stated that the voltage measurement of every single cell in a series connection is inevitable, whereas it is possible depending on the architecture - to monitor not all temperatures in a module. For the temperature monitoring in series production it is also imaginable to combine the actual measurement of temperatures, that is needed for the load management, with bimetall switches that shut down the pack independently by a safety circuit in case of an overtemperature. This would lead to only a few temperature sensors per pack and therefore reduced costs. The positioning and needed amount of sensors can be simulated beforehand. For the thermal management it could be shown that there are driving applications such as fast charge where cooling is mandatory. Therefore, a liquid cooling system in the ground plate was designed and taken into operation. A possibility to connect the cells to the cooling system was given by the aluminum plates. With the designed cooling system it was possible to reduce the temperature gradient within the pack and the cells below $2.5^{\circ} \mathrm{C}$ which is of great importance for an even aging of the pack. Still, further cooling concepts such as a direct cooling of the contacts can be considered to reduce the overall weight of the system.

\section{Acknowledgments}

This work has been kindly supported by the German Ministry of Economics and Technology (BMWi) within the project Smart Wheels.

\section{References}

[1] IEA Statistics $\mathrm{CO}_{2}$ Emissions From Fuel Combustion 2011 - Highlights, Paris, IEA Publications, 2011. 
[2] Tescon, http://www.tecson.de/, accessed on 2012-01-15.

[3] A. Dinger et.al., Batteries for Electric Cars Challenges, Opportunities and the Outlook to 2020, Boston Consulting Group, 2010.

[4] J. Vetter et al., Aging mechanisms in lithium-ion batteries, Journal of Power Sources, 146(2005), 269-281.

[5] P. Sinhuber et.al., Conceptional Considerations for Electrification of Public City Buses - Energy Storage System and Charging Stations, Emobility - Electrical Power Train, 2010, 1-5.

[6] Kokam, http://www.kokam.com/, accessed on 2012-01-17.

[7] DIN EN 60529, http://www.vde-verlag.de/, accessed on 2012-01-17.

[8] Ch. J. Orendorff Mitigating Catastrophic Failure in Lithium-Ion Batteries, 978-1-59430-217-6, Battery Safety - Advances in System Design, Integration \& Testing for Safety \& Reliability , 2010, 88-109.

[9] M Broussely Aging mechanism in Li ion cells and calendar life predictions, Journal of Power Sources, 97-98 (2001), 13-21.

\section{Authors}

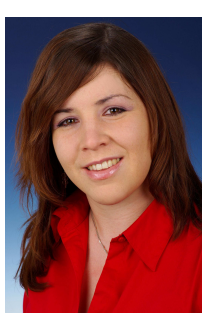

Susanne Rothgang received her diploma in Electrical Engineering from Friedrich-Alexander University Erlangen-Nuremberg in 2010. In June 2010 she joined ISEA as a research associate. Her areas of interest are $\mathrm{HV}$ batteries and the related vehicle integration with a special focus on safety aspects.

Benedikt Lunz received his diploma in Electrical Engineering from Friedrich-Alexander Univer-


sity Erlangen-Nuremberg in 2008. In September 2008 he joined ISEA as a research associate. His areas of interest are charging strategies for electric vehicle batteries and the grid integration of stationary storage systems.

Dirk Uwe Sauer received his diploma in Physics from University of Darmstadt in 1994 and the Ph.D. degree from Ulm University, Ulm, Germany, in 2003. From 1992 to 2003, he was a Scientist at Fraunhofer Institute for Solar Energy Systems, Freiburg, Germany. In October 2003, he was a Junior Professor, and since 2009 he has been a Professor at RWTH Aachen University, Aachen, Germany, for Electrochemical Energy Conversion and Storage Systems. 